\title{
A Study on the Effects of Polite Yawning Technique on Rehabilitation of Olfaction After Total Laryngectomy
}

\author{
Ehsan Khadivi (iD ${ }^{1}$, Kamran Khazaeni ${ }^{1}$, Leila Vazifeh Mostaan ${ }^{2}$, Maryam Salehi ${ }^{1}$, Iraj Vakili ${ }^{3}$, Seyed \\ Alireza Javadinia (ii) ${ }^{4}$ and Ahmadreza Ahmadi (iD ${ }^{3, *}$ \\ ${ }^{1}$ Sinus and Surgical Endoscopic Research Center, Mashhad University of Medical Sciences, Mashhad, Iran \\ ${ }^{2}$ Cancer Research Center, Mashhad University of Medical Sciences, Mashhad, Iran \\ ${ }^{3}$ Student Research Committee, Mashhad University of Medical Sciences, Mashhad, Iran \\ ${ }^{4}$ Cellular and Molecular Research Center, Sabzevar University of Medical Sciences, Sabzevar, Iran \\ "Corresponding author: Head and Neck Surgery Ward, Emam Reza Hospital, Emam Reza Sq, Mashhad, Iran. Email: ahmadreza.md@gmail.com
}

Received 2019 November 16; Revised 2020 June 15; Accepted 2020 June 20.

\begin{abstract}
Background: Laryngeal cancer is one of the most common head and neck cancers for which total laryngectomy is the preferred treatment in advanced stages. Major disabilities of this surgical procedure include loss of voice and nasal function, swallowing difficulties, and psychological consequences.

Objectives: This study aimed to investigate the effect of the Polite Yawning technique on olfactory quality in patients undergoing total laryngectomy.

Methods: In this cross-sectional study, after total laryngectomy, the patients with olfactory dysfunction were enrolled and the quality of olfaction was evaluated by the Quick Odor Detection test before and after receiving the Nasal Airflow Inducing Maneuver technique training. Statistical analysis was performed using SPSS 21 software by the Wilcoxon or McNemar's test. The significance level was considered for $\mathrm{P} \leq 0.05$.

Results: A total of 40 patients were evaluated. The mean age was $55.98 \pm 6.27$ years. Eighty-five percent of the patients were male (n =34). The difficulty score in learning maneuvering was $2.5 \pm 1.3$, and $90 \%$ had no problem with learning it. Before maneuver, none of the patients had normal olfaction and had hyposmia mostly $(n=32,80 \%)$. Immediately after the maneuver, there was a sensation of smell in all patients and the rate of hyposmia in patients was reduced to $65 \%(\mathrm{P}=0.008)$. Normal olfactory status was reported in half of the patients one month after the maneuver (21 patients, $52.5 \%, \mathrm{P}=0.0001)$, and there was a sense of smell in all patients $(\mathrm{P}=$ $0.0001)$.

Conclusions: The results proved that olfactory quality can be rehabilitated after laryngectomy by the nasal airflow-inducing maneuver (the "Polite Yawning" technique). It is a patient-friendly method; however, a single training session is probably insufficient and most patients may need more training sessions.
\end{abstract}

Keywords: Olfaction, Smell, Laryngectomy, Polite Yawning Technique, Laryngeal Cancer

\section{Background}

Laryngeal cancer is one of the most common cancers of the head and neck region (1). The preferred treatment for laryngeal cancer in advanced stages is total laryngectomy, which is associated with disabilities, the most common being vocal disorder. More important from among the complications are nasal dysfunction, weak cough, difficulty in swallowing, and psychological consequences (2). One of the most important outcomes of total laryngectomy is olfactory dysfunction (3). To improve the olfactory function in patients undergoing laryngectomy, extensive efforts have been made, primarily to enhance the nasal airflow as a means of delivering aromatic molecules to olfac- tory neuroepithelium (4).

Two techniques have been employed so far: the use of a temporary device that restores the connection between the nose/mouth and the lower airways (prosthetic procedures) (5), and the use of oro-facial muscles to create nasal airflows (behavioral intervention) $(4,6)$. To improve the olfactory quality of patients, one behavioral technique called the nasal airflow inducing maneuver (NAIM) has proved more practical. This method often referred to as the Polite Yawning technique, was first described by Hilgers et al. (7), NAIM is aimed to create a negative pressure gradient in the oropharyngeal cavity through which the air enters the nasal cavity and whereby the orthonasal olfaction is re- 
stored. The success rate of these methods in the improvement of the olfactory sense of patients after total laryngectomy varies from $46 \%$ to $88 \%$ (7-9). The treatment procedure and its sequence vary in different studies. Nevertheless, there is a consensus that this method can significantly improve the olfaction of patients (6).

To stimulate the olfactory epithelium, there are two pathways including the orthonasal pathway (the entry of the odorant through the nasal cavity) and the retronasal pathway (the entry of the odorant molecule through the nasopharynx and posterior concha). The olfactory sense and in turn, the sense of taste, is substantially reliant on the olfactory epithelium stimulation through the nose, and therefore, any dysfunction in the olfaction is closely associated with disorders in the taste sensation $(10,11)$.

There are two general theories regarding the pathogenesis of olfactory disorder in patients undergoing total laryngectomy. The first theory considers the impaired nasal flow as the cause of olfactory disorder in these patients, which leads to the absence of aromatic molecules (to stimulate) into olfactory mucus $(6,12-15)$. As the airflow decreases, histological changes involve specific heterogeneity and the formation of degeneration regions (16). Mucosal secretion decreases after laryngectomy. Yet, despite the increased number of celiac cells, mucociliary clearance of the epithelial cells does not happen $(4,17)$. The second theory states that the main cause of olfactory disorder in patients undergoing laryngectomy resides with laryngeal nerve damage, although most researchers support the first theory (18).

Studies by Ward et al. (19), Haxel et al. (20), Hilgers et al. (21), and Risberg-Berlin et al. (9). have shown that application of NAIM in patients undergoing laryngectomy can improve the patients' sense of smell, and it is easy for the patients to learn and apply it on a daily basis.

\section{Objectives}

According to the literature, no study seems to have been conducted in Iran on the efficacy of behavioral interventions to improve the sense of smell of patients after total laryngectomy. Therefore, the present study was conducted to determine the effect of polite yawning on olfactory quality in patients undergoing total laryngectomy.

\section{Methods}

This study was a cross-sectional study. In this study, the Polite Yawning technique was initially trained to the patients undergoing total laryngectomy, and the patients were assessed for olfactory status by the Quick
Odor Detection Test and a semi-structured questionnaire on the quality of olfactory, taste, and appetite. The research protocol was approved at the Ethics Committee of Mashhad University of Medical Sciences (identifier: IR.MUMS.fm.REC.1395.266). Written informed consent was obtained from the patients.

Inclusion criteria were (1) patients undergoing total laryngectomy; (2) ability to speak in Persian; (3) adequate intelligence for understanding the technique; and (4) consent for participation. Initial exclusion criteria were: (1) olfactory disorder before laryngectomy; (2) olfactory disorder with larynx bypass; (3) maxillary anatomical changes that prevent the maneuver; (4) radiation-induced fibrosis; (5) weakness of one or double-sided lower lip that prevents from complete closure; (6) history of pre-surgical head trauma; (7) history of respiratory infections with olfactory dysfunctioning complications such as chronic rhinosinusitis; (8) nasal cavity cancer; (9) recurrence of the primary tumor; and (10) chronic medical conditions such as diabetes mellitus, hypertension, and ischemic heart disease. Secondary exclusion criteria included learning to conduct maneuvers in two sessions of education, and failure to visit for olfactory status follow-up.

During the first preoperative visit, all the participants underwent flexible laryngoscopy for anatomical disorders affecting the nasal airflow. The patients were also evaluated for impaired facial and jaw disorders. In case these were normal, the patients were evaluated for the absence of olfactory neuropathy such that after larynx bypass insertion and airflow establishment in the nose, the sense of smell was evaluated using an aromatic substance known by the patient.

The olfactory evaluation was performed by a semistructured questionnaire on the quality of olfactory, taste and, appetite together with the Quick Odor Detection test through interviews. According to the results of the olfactory diagnostic test, the patients were categorized to have either olfactory disorder: patients with normal olfaction versus patients with abnormal olfaction (hyposmia and anosmia), or olfactory sensation: patients with olfactory sensation (natural sense of smell and hyposmia) versus patients lacking the sense of smell (anosmia). The Quick Odor Detection test was carried out using the kits made by the Saba Medical Engineering Company of Sabalan, in which each kit consisted of six brown strips with different aromatic substances. Each patient was evaluated respectively from the first strip to the sixth one. In this way, upon the patient's readiness, a few lines were scratched on the brown strip with a pencil, and the tape was immediately placed before the patient's nose. The patient was then asked to select an option that corresponded to the smelled sensation 
(if the patient was not able to read the options, the options were read out aloud in sequence for him/her). In case the patients were not able to properly diagnose all the olfactory variables, the patient was classified as anosmic. If the five items were correctly identified, the person would be classified as normosmia. If the patient was within these two ranges, s/he would be classified as hyposmia. Assessment occasions were involved pre-operative, before the intervention, within the first week after intervention, and one month after the intervention.

All the patients received NAIM technique training. Through the maneuver, the patients close their lips and imitate yawning politely by lowering the jaw, tongue and its base, the floor of the mouth, and the soft palate. This action increases oropharyngeal volume and allows airflow into the nose. By creating an orthonasal rejuvenation stream, the olfactory stimulants can reach the olfactory receptors and create a sense of smell. The technique was orally trained along with images and videos at the first post-operative visit. The patients were encouraged to repeat the maneuver on a number of occasions during the session as well as during daily activities. The fundamentals of the technique as well as its use were periodically recalled in the course of patient visits. A water manometer was used to assess the correct maneuvering of the patient. Also, a manometer with written instructions was given to the patients to use at home and to provide biofeedback.

Data was collected by SPSS 21 software. Both descriptive analysis (frequency, percentage, mean, and standard deviation) and inferential analysis (chi-square test and Fisher's exact test) were performed. P values less than 0.05 were considered significant.

\section{Results}

A total of 53 patients were candidates for enrollment in the study of whom three failed to enter. Of the 50 patients recruited in the study, one died during follow-up, four had no follow-up, three had tumor recurrence, and two had long-term hospitalization, and were therefore excluded, so 40 patients were left for final assessment. The mean age of the studied patients was $55.98 \pm 6.27$ years, and they were mostly over 56 years old (55\%). In this study, only $15 \%$ of the patients had not received adjuvant therapy. The mean time of surgery in patients under study was $1.74 \pm 1.88$ years. Table 1 displays the demographic data of the patients.

The patients were asked about the difficulty in learning the maneuver based on a scale of zero (no difficulty) to 10 (very difficult), revealing a mean difficulty score of $2.5 \pm 1.3$ with $90 \%$ having no problems learning it. The median for training times was four.

\begin{tabular}{ll}
\hline Table 1. Demographic Characteristics of the Participants & \\
\hline Variable & Values \\
\hline Gender & \\
Male & $34(85)$ \\
\hline Female & $6(15)$ \\
\hline Age group & \\
\hline 255 years & $22(55)$ \\
$<55$ years & $18(45)$ \\
\hline Education level & $17(42.5)$ \\
\hline Illiterate & $17(42.5)$ \\
\hline Primary school & $6(15)$ \\
\hline Higher levels
\end{tabular}

${ }^{\mathrm{a}}$ Values are expressed as No. (\%).

The Cochran test was used to compare the frequencies of olfactory quality, olfactory sensation, and olfactory disorder before, immediately after, and one month after intervention. The results showed that the three variables significantly improved after intervention $(\mathrm{P}<0.0001)$. Table 2 compares the frequency of olfactory quality, the sense of smell, and the presence of olfactory disorder before, immediately after, and one month after intervention in the studied patients.

\section{Discussion}

The aim of this study was to investigate the effect of the polite yawning on olfaction in patients undergoing total laryngectomy. In this section, we first mention the most significant findings. In the next step, the findings of the study are compared with the results of similar studies, and explanations concerning the existing differences are described.

Overall, the results of this study showed that using NAIM or the Polite Yawning technique can significantly improve the olfactory sensation of patients after total laryngectomy. The study of Hilgers et al. (7), on 44 patients undergoing laryngectomy, behavioral intervention is associated with improved olfaction disorder. This finding is consistent with the current study. Also, the study of RisbergBerlin et al. (8), which was conducted to investigate olfactory rehabilitation after total laryngectomy in Swedish patients, showed that the NAIM technique was effective in improving the olfactory disorder in the patients, in line with our study. Also, Risberg-Berlin et al. (8) conducted another study on the efficacy of the NAIM technique in improving the olfactory quality of patients after total laryngectomy, 


\begin{tabular}{|c|c|c|c|c|}
\hline \multirow{2}{*}{ Point Quality } & \multicolumn{4}{|c|}{ Analysis Time } \\
\hline & Before Intervention & $\begin{array}{l}\text { Immediately After } \\
\text { Intervention }\end{array}$ & $\begin{array}{l}\text { One Month After } \\
\text { Intervention }\end{array}$ & Test Result \\
\hline Anosmia & $8(20)$ & $0(0)$ & $0(0)$ & \multirow{3}{*}{$\mathrm{Q}=45.0 ; \mathrm{P}=0.001$} \\
\hline Hyposmia & $32(80)$ & $26(65)$ & $19(47.5)$ & \\
\hline Normal & $0(0)$ & $14(35)$ & $21(52.5)$ & \\
\hline Having a sense & & & & $Q=16.0 ; P=0.001$ \\
\hline Yes & $32(80)$ & $40(100)$ & $40(100)$ & \\
\hline No & $8(20)$ & $0(0)$ & $0(0)$ & \\
\hline
\end{tabular}

${ }^{\mathrm{a}}$ Values are expressed as No. (\%).

and on olfactory rehabilitation using Polite Yawning technique. Consistent with our findings, the results confirmed that NAIM is an easy method for patients, which is not only cost-effective but also efficient in improving the olfactory function of patients undergoing laryngectomy (8). Recently, Ward et al.'s study (19), showed that both groups reported significantly improved olfactory abilities. However, the improvement rate before and immediately after intervention was significantly higher in the clinician assisted group, although the effects of these methods are observed only in short-term periods, contrary to Risberg-Berlin et al.'s study (19). In Haxel et al.'s study (20), the effect of NAIM on olfactory rehabilitation was evaluated in patients with laryngectomy, and the results showed that the NAIM technique was a successful method in olfactory rehabilitation in patients undergoing laryngectomy.

The study of Ward et al. (19) demonstrated that the use of NAIM in patients undergoing laryngectomy has resulted in improved or recovered sense of smell in $90.24 \%$ of the patients (22). The most important factors associated with improving the sense of smell in patients after total laryngectomy, especially the sustainability of its long-term results, are the use of these techniques during daily activities as well as willingness to perform the technique. On the other hand, the main reasons for non-use of the maneuver comprise of (1) ineffectiveness on the improvement of the person's sense of smell; (2) forgetting the way to perform the maneuver; (3) olfactory recovery without performing the maneuver; and (4) insignificance of smell for the individual. Overall, there is a significant relationship between the sense of smell and the proper performance of the maneuver (21).

Using NAIM or Polite Yawning technique in patients with larynx cancer undergoing total laryngectomy creates a negative pressure gradient in the oropharyngeal cavity through which the air enters the nasal cavity and reestablishes the orthonasal smell. The success rate of these methods in improving the olfactory power of patients undergoing total laryngectomy varies from $46 \%$ to $88 \%$ (7-9). The treatment procedure and its sequence vary in different studies. Nonetheless, there is a general consensus that this method can significantly improve the olfaction ability of patients (6).

Also, the results of this study showed that learning the NAIM or Polite Yawning technique is easy and achievable within a reasonable period of time. Similar to the current study, Risberg-Berlin et al. (9) concluded that while one-session interventions have significant efficacy, some patients require more sessions to learn the technique. Hilgers et al.'s study (7) indicated that NAIM is trainable to all patients and could be trained to a patient in one session within 30 minutes. In the study of Haxel et al. (20), all patients (100\%) had a great interest in learning NAIM; $90 \%$ had no problems learning it and were eager both use the maneuver in daily activities and to recommend its use to other patients.

\subsection{Conclusions}

The results of this study showed that the use of the Polite Yawning technique has significant positive effects on improving the sense of smell of patients undergoing total laryngectomy. Considering the simplicity of learning this method, it is recommended for surgeons to provide relevant education to patients in this regard. It should be noted, that the correct and consistent use of this technique requires reminding and practicing at each visit. The main strength of this research is the attempt to improve the olfactory quality in patients undergoing laryngectomy in the Iranian population, which is among the first ones. The use of the Quick Odor Detection Test, which is a precise method for assessing the sense of smell in patients, is a further strength of the study.

This study had also some limitations which included lack of differentiation between the senses of taste and 
smell, the lack of clarity concerning the effect of this maneuver on the patients' quality of life, and the relatively small sample size that prevents the generalizability of the findings. Future studies with larger sample sizes and parallel groups are suggested. It is also recommended that future studies be carried out by distinguishing the olfactory and taste quality, as well as their impact on the patients' quality of life.

\section{Footnotes}

Authors' Contribution: Study concept and design: EK, and IV. Analysis and interpretation of data: SAJ, and AA. Drafting of the manuscript: MS. Critical revision of the manuscript for important intellectual content: SAJ, KK, and LV. Statistical analysis: MS.

Conflict of Interests: There are no conflicts of interest.

Ethical Approval: The research protocol was approved at the Ethics Committee of Mashhad University of Medical Sciences (identifier: IR.MUMS.fm.REC.1395.266).

Funding/Support: This thesis research was conducted by Iraj Vakili on the basis of research project no.: 950097, and is sponsored by the Deputy of Research and Technology of Mashhad University of Medical Sciences.

Informed Consent: Necessary and clear explanations were provided to all patients, and if they agreed to participate, written consent was obtained from the patients.

\section{References}

1. Curado MP, Hashibe M. Recent changes in the epidemiology of head and neck cancer. Current opinion in oncology. 2009;21(3):194-200. doi: 10.1097/CCO.0b013e32832a68ca. [PubMed: 19363341].

2. Pawar PV, Sayed SI, Kazi R, Jagade MV. Current status and future prospects in prosthetic voice rehabilitation following laryngectomy. Journal of cancer research and therapeutics. 2008;4(4):186. doi: 10.4103/0973-1482.44289. [PubMed: 19052392].

3. Risberg-Berlin B. Olfactory function after total laryngectomy in Swedish patients after rehabilitation with the Nasal Airflow-Inducing Maneuver. Institute of Clincial Sciences. Department of Otorhinolaryngology; 2009.

4. Moor JW, Rafferty A, Sood S. Can laryngectomees smell? Considerations regarding olfactory rehabilitation following total laryngectomy. The Journal of Laryngology \& Otology. 2010;124(4):361-5. doi: 10.1017/S0022215109992489. [PubMed: 20059791].

5. Göktas O, Fleiner F, Paschen C, Lammert I, Schrom T. Rehabilitation of the olfactory sense after laryngectomy: Long-term use of the larynx bypass. Ear Nose Throat J. 2008;87:528-30. doi: 10.1177/014556130808700913. [PubMed: 18800327].

6. Ward E, Rumbach A, van As-Brooks CJ. Olfaction following total laryngectomy. Journal of Laryngology and Voice. 2012;2(1):10. doi: 10.4103/2230-9748.94728.
7. Hilgers FJ, van Dam FS, Keyzers S, Koster MN, van As CJ, Muller MJ. Rehabilitation of olfaction after laryngectomy by means of a nasal airflowinducing maneuver: the polite yawning technique. Archives of Otolaryngology-Head \& Neck Surgery. 2000;126(6):726-32. doi: 10.1001/archotol.126.6.726. [PubMed: 10864109].

8. Risberg-Berlin B, Möller RY, Finizia C. Effectiveness of olfactory rehabilitation with the nasal airflow-inducing maneuver after total laryngectomy: one-year follow-up study. Archives of Otolaryngology-Head \& Neck Surgery. 2007;133(7):650-4. doi: 10.1001/archotol.133.7.650. [PubMed: 17638776].

9. Risberg-Berlin B, Ylitalo R, Finizia C. Screening and Rehabilitation of Olfaction After Total Laryngectomy in Swedish Patients: Results From an Intervention Study Using the Nasal Airflow-Inducing Maneuver. Archives of Otolaryngology-Head \& Neck Surgery. 2006;132(3):301-6. doi: 10.1001/archotol.132.3.301. [PubMed: 16549751].

10. Hočevar-Boltežar I, Mumović G. Olfaction and gustation abilities after total laryngectomy. Radiology and Oncology. 2014;48(3). doi: 10.2478/raon-2013-0070. [PubMed: 25177245].

11. Mumovic G, Hocevar-Boltezar I. Olfaction and gustation abilities after a total laryngectomy. Radiology and oncology. 2014;48(3):301-6. doi: 10.2478/raon-2013-0070. [PubMed: 25177245].

12. Moore-Gillon V. The nose after laryngectomy. Journal of the Royal Society of Medicine. 1985;78(6):435-9. doi: 10.1177/014107688507800603. [PubMed: 3999077].

13. Mozell MM, Hornung DE, Leopold DA, Youngentob SL. Initial mechanisms basic to olfactory perception. American journal of otolaryngology. 1983;4(4):238-45. doi:10.1016/S0196-0709(83)80065-9.

14. Ritter FN. Fate of olfaction after laryngectomy. Archives of Otolaryngology-Head \& Neck Surgery. 1964;79(2):169. doi: 10.1001/archotol.1964.00750030175013. [PubMed:14086478].

15. Van Dam FS, Hilgers FJ, Emsbroek G, Touw FI, Van As CJ, De Jong N. Deterioration of olfaction and gustation as a consequence of total laryngectomy. The Laryngoscope.1999;109(7):1150-5. doi:10.1097/00005537199907000-00027. [PubMed: 10401859].

16. Miani C, Ortolani F, Bracale AMB, Petrelli L, Staffieri A, Marchini M. Olfactory mucosa histological findings in laryngectomees. $\mathrm{Eu}$ ropean archives of oto-rhino-laryngology. 2003;260(10):529-35. doi: 10.1007/s00405-003-0638-3. [PubMed: 12835945].

17. Fisher EW, Lund VJ, Rutman A. The human nasal mucosa after deprivation of airflow: a study of laryngectomy patients. Rhinology. 1992;30(1):5-10. doi: 10.1016/S0140-6736(68)90647-8.

18. Henkin R, Hoye RC, Ketcham AS, Gould WJ. Hyposmia following laryngectomy. The Lancet. 1968;292(7566):479-81. doi: 10.1016/S01406736(68)90647-8.

19. Ward E, Coleman A, As-Brooks V, Kerle S. Rehabilitation of olfaction post-laryngectomy: a randomised control trial comparing clinician assisted versus a home practice approach. Clinical Otolaryngology. 2010;35(1):39-45. doi: 10.1111/j.1749-4486.2009.02050.x. [PubMed: 20447161].

20. Haxel BR, Fuchs C, Fruth K, Mann WJ, Lippert BM. Evaluation of the efficacy of the 'nasal airflow-inducing manoeuvre'for smell rehabilitation in laryngectomees by means of the Sniffin' Sticks test. Clinical Otolaryngology. 2011;36(1):17-23. doi: 10.1111/j.1749-4486.2011.02261.x. [PubMed: 21244643].

21. Hilgers FJ, Jansen HA, van As CJ, Polak MF, Muller MJ, van Dam FS. Long-term Results of Olfaction Rehabilitation Using the Nasal Airflow-Inducing (Polite Yawning) Maneuver After Total Laryngectomy. Archives of Otolaryngology-Head \& Neck Surgery. 2002;128(6):648-54. doi: 10.1001/archotol.128.6.648. [PubMed: 12049558].

22. Morales-Puebla JM, Morales-Puebla ÁF, Jiménez-Antolín JA, MuñozPlatón E, Padilla-Parrado M, Chacón-Martínez J. Olfactory rehabilitation after total laringectomy. Acta Otorrinolaringologica (English Edition). 2010;61(2):128-34. doi:10.1016/S2173-5735(10)70020-X. 